\title{
Erratum: Single-neuron RNA-Seq: technical feasibility and reproducibility
}

\section{Shenfeng Qiu ${ }^{1,2}$, Shujun Luo ${ }^{3}$, Oleg Evgrafov ${ }^{1}$, Robin Li $^{3}$, Gary P. Schroth ${ }^{3}$, Pat Levitt ${ }^{1,2}$, James A. Knowles ${ }^{1,4 *}$ and Kai Wang ${ }^{1,4 *}$}

1 Zilkha Neurogenetic Institute, University of Southern California, Los Angeles, CA, USA

${ }^{2}$ Department of Cell and Neurobiology, University of Southern California, Los Angeles, CA, USA

3 Illumina, Inc., Hayward, CA, USA

${ }^{4}$ Department of Psychiatry, University of Southern California, Los Angeles, CA, USA

*Correspondence:knowles@med.usc.edu; kaiwang@usc.edu

\section{Edited by:}

Paul Pavlidis, University of British Columbia, Canada

\section{A commentary on}

Single-neuron RNA-Seq: technical feasibility and reproducibility

by Qiu, S., Luo, S., Evgrafov, O., Li, R., Schroth, G. P., Levitt, P., Knowles, J. A., and Wang, K. (2012). Front. Gene. 3:124. doi: 10.3389/fgene.2012.00124

To the Editor:

In our manuscript entitled "Singleneuron RNA-Seq: technical feasibility and reproducibility," published online on July 6th, 2012, we have made a few serious mistakes which need to be corrected:

We referenced several points and quoted statements from a recent study by Okaty et al., but we neither cited the paper nor placed quotation marks on the statements. The full citation should be "Okaty, B. W., Sugino, K., and Nelson, S. B. (2011). Cell type-specific transcriptomics in the brain. J. Neurosci. 31, 6939-6943."

We request to add a citation after "The analysis of each single-cell transcriptome consists of several independent steps" in the first paragraph of Results, as "The analysis of each single-cell tran- scriptome consists of several independent steps (Okray et al., 2011)," given that these points were previously raised by Okaty et al. We request to add a citation to the first paragraph of Introduction, as "studies of gene expression and function in the brain were restricted to a relatively small number of genes (Luo and Geschwind, 2001; Zhang et al., 2002; Okaty et al., 2011)," given that this points was recently reviewed in the Okaty et al., paper. More importantly, we have made a mistake in quoting sentence from Okray et al., without quotation or citation. We wish to change the following sentence:

Moreover, gene expression may be regulated in opposing directions in different cell types, thereby appearing static in composite data.

To

Moreover, as previously discussed by Okaty et al., "gene expression may be regulated in opposing directions in different cell types, thereby appearing static in composite data (Okaty et al., 2011).”

We apologize to the readers of Frontiers in Genetics and to the authors of the Okaty et al., manuscript, for our failure to correct these mistakes during the review process of our manuscript.

\section{REFERENCES}

Luo, Z., and Geschwind, D. H. (2001). Microarray applications in neuroscience. Neurobiol. Dis. 8, 183-193.

Okaty, B. W., Sugino, K., and Nelson, S. B. (2011). Cell type-specific transcriptomics in the brain. J. Neurosci. 31, 6939-6943.

Zhang, W., Wang, H., Song, S. W., and Fuller, G. N. (2002). Insulin-likegrowth factor binding protein 2: gene expression microarrays and the hypothesis-generation paradigm. Brain Pathol. 12, 87-94.

Received: 09 January 2013; accepted: 12 February 2013; published online: 27 February 2013.

Citation: Qiu S, Luo S, Evgrafov O, Li R, Schroth GP, Levitt P, Knowles JA and Wang K (2013) Erratum: Single-neuron RNA-Seq: technical feasibility and reproducibility. Front. Genet. 4:23. doi: 10.3389/fgene. 2013.00023

This article was submitted to Frontiers in Neurogenomics, a specialty of Frontiers in Genetics.

Copyright (c) 2013 Qiu, Luo, Evgrafov, Li, Schroth, Levitt, Knowles and Wang. This is an open-access article distributed under the terms of the Creative Commons Attribution License, which permits use, distribution and reproduction in other forums, provided the original authors and source are credited and subject to any copyright notices concerning any third-party graphics etc. 